\title{
NOTAS
}

\section{Rendimiento de un sistema de rotación corta de alta densidad con Salix spp. en Buenos Aires, Argentina}

\author{
Yield of a short rotation coppice plantation of Salix spp. in Buenos Aires, Argentina
}

\author{
Guillermo N Doffo a , Fabio G Achinelli ${ }^{\text {a,b }}$, María E Rodríguez a, Virginia MC Luquez ${ }^{\text {a* }}$ \\ *Autor de correspondencia: ${ }^{a}$ Universidad Nacional de La Plata (UNLP) - Consejo Nacional de Investigaciones Científicas y Técnicas \\ (CONICET), CC 327, 1900 La Plata, Argentina, tel.: +54-221-423-6618, fax +54-221-423-3698, vluquez@agro.unlp.edu.ar \\ ${ }^{\mathrm{b}} \mathrm{CIC}$, Buenos Aires, Argentina.
}

\begin{abstract}
SUMMARY
In Argentina, energy production depends mostly on fossil fuels; hence it is desirable to diversify the energy sources including renewable fuels such as forest biomass. A drawback for this development is the lack of information about the productivity of short rotation coppice systems (SRC) under local conditions. Willows are used in several countries as a source of biomass for energy, although in Argentina the information about yield at local level is scarce. An SRC system was established in agricultural soil in Buenos Aires Province, Argentina. The aim of this work was to estimate the effects of irrigation, plantation density and genotype on biomass production. The clones used were the hybrid Salix matsudana $x$ Salix alba 'Barrett 13-44 INTA' and the open pollinated Salix alba 'Yaguareté INTA - CIEF'. There were no statistically significant effects of clone and plantation density on biomass productivity. The only significant factor was irrigation, which explained 83 to $92 \%$ of yield variability. The dry biomass yields are similar to those reported elsewhere for rainfed SRC willows, ranging between 11.1 and $22.6 \mathrm{Mg} \mathrm{ha}^{-1}$ year $^{-1}$.
\end{abstract}

Key words: willows, dendroenergy, biomass.

\section{RESUMEN}

La matriz energética de Argentina depende principalmente de los combustibles fósiles, por lo que resulta deseable diversificarla, incluyendo fuentes renovables como la biomasa forestal. Una limitación para este desarrollo es la falta de información sobre la productividad de sistemas de rotación corta de alta densidad (SRC) destinados a la producción de biomasa en condiciones locales. El objetivo de este trabajo fue evaluar el efecto del genotipo, la densidad de plantación y la disponibilidad hídrica sobre la productividad de un sistema SRC con sauces en un suelo agrícola en la provincia de Buenos Aires. La principal limitación para la productividad es la disponibilidad de agua, que explica entre el 83 y el $92 \%$ de la variabilidad en la biomasa seca, mientras que no hubo efectos significativos del genotipo y la densidad de plantación. Los rendimientos de biomasa seca obtenidos en este ensayo son comparables a los reportados para sistemas SRC en otros países, variando entre 11,1 y $22,6 \mathrm{Mg} \mathrm{ha}^{-1}$ año $^{-1}$.

Palabras clave: sauce, dendroenergía, biomasa.

\section{INTRODUCCIÓN}

En Argentina, las energías renovables representan el $9,4 \%$ de las fuentes primarias, correspondiendo un $5 \%$ a la biomasa (Ministerio de Energía y Minería 2016). Los sauces (Salix spp.) se utilizan en diversos países como cultivo bioenergético, en sistemas basados en el corte y rebrote de plantas madres, con elevadas densidades de plantación y corta rotación (máximo 4 años), denominados SRC (Short Rotation Coppice, Keoleian y Volk 2005). Una importante limitación para el desarrollo de la dendroenergía en Argentina es la escasez de información sobre la productividad de los cultivos leñosos bioenergéticos a nivel local. Las Salicáceas constituyen el tercer cultivo forestal con una superficie de 97.893 ha (Cerrillo 2014), pero no existen plantaciones comerciales con el objetivo primario de producir biomasa para energía.

La relación de la densidad de plantación con la productividad en sistemas SRC de sauce es afectada por la longitud de la rotación y el genotipo (Willebrand et al. 1993). Otro factor importante es la disponibilidad hídrica, ya que los sauces son sensibles a la sequía (Wikberg y Ögren 2007). En muchas zonas de Argentina es común la existencia de períodos de estrés hídrico durante el verano (Hurtado et al. 2006), que podrían limitar la productividad de los sauces plantados en SRC. Sin embargo, algunos 
genotipos pueden aclimatarse a la sequía, reduciendo la apertura estomática y el área foliar, e incrementando la tolerancia a la cavitación (Wikberg y Ögren 2007). Esta variabilidad genética podría explotarse para obtener clones de sauce con mayor tolerancia al estrés hídrico.

Los clones utilizados en este trabajo son Salix matsudana Koidz. x Salix alba L. 'Barrett 13-44 INTA' (abreviado Barrett) y Salix alba L. 'Yaguareté INTA-CIEF' (abreviado Yaguareté). Si bien estos clones no fueron seleccionados como cultivo bioenergético, presentan crecimiento rápido y fuste recto, características deseables en genotipos destinados a producir biomasa (Karp et al. 2011). Yaguareté es más susceptible a la sequía que Barrett debido a su mayor sensibilidad a la cavitación (Doffo et al. 2017), mientras que Barrett tiene escasa tolerancia a períodos largos de inundación (Cerrillo et al. 2013). Ambos clones tienen rendimientos similares creciendo a campo en rotaciones largas (Cerrillo 2014) pero se desconoce su potencialidad productiva en un sistema SRC.

El objetivo de este trabajo es evaluar los efectos de la disponibilidad hídrica y la densidad de plantación sobre la productividad en un sistema SRC de dos clones de sauce con respuestas contrastantes frente a la inundación y la sequía. La hipótesis es que los factores analizados (genotipo, densidad de plantación y disponibilidad hídrica) afectarán la producción de biomasa.

\section{MÉTODOS}

Diseño experimental. Los clones se obtuvieron en programas de mejoramiento del INTA (Instituto Nacional de Tecnología Agropecuaria). Barrett es un híbrido de Salix matsudana x Salix alba, y Yaguareté es hijo de polinización abierta de una madre S. alba (Cerrillo 2014).

El ensayo se instaló en la ciudad de La Plata (3459'09" $\mathrm{S} ; 57^{\circ} 59^{\prime} 42^{\prime}$ ' O) en un suelo Argiudol típico (Hurtado et al. 2006). Previo a la plantación, se tomó una muestra compuesta de suelo ( $25 \mathrm{~cm}$ superiores del perfil) para determinar su fertilidad (cuadro 1). La clase textural se determinó por el método de Bouyucus, indicando un suelo franco limoso (26,2 \% de arcillas, $46 \%$ de limo y $27,8 \%$ de arena).

El diseño experimental fue en bloques con parcelas divididas, con tres factores: riego, densidad y clon. Los tres bloques permitieron excluir la variabilidad causada por la pendiente presente en el sitio de ensayo. Cada bloque fue dividido en dos parcelas principales (con riego y sin riego, primer factor). Las parcelas fueron separadas entre sí por calles de cuatro metros de ancho, para evitar el movimiento lateral de agua hacia las parcelas de secano. Cada parcela se dividió en dos subparcelas con diferentes densidades de plantación (segundo factor), las densidades fueron 13.333 plantas ha ${ }^{-1}$ (configuración de $1 \mathrm{~m}$ por $0,75 \mathrm{~m}$ ) y 20.000

Cuadro 1. Características del suelo al comienzo del ensayo.

Characteristics of top soil at the start of the plantation.

\begin{tabular}{|c|c|}
\hline Variable & Valor inicial \\
\hline pH (pehachímetro) & 6,63 \\
\hline Materia orgánica (\%, Método de Walkey-Black) & 2,8 \\
\hline Carbono total (\%, método de Walkey-Black ) & 1,63 \\
\hline Nitrógeno total (\%, método de macro Kjeldahl) & 0,12 \\
\hline Relación C:N & 13,2 \\
\hline Fósforo (Bray-I) (mg kg ${ }^{-1}$, espectrofotometría) & 20,9 \\
\hline Conductividad eléctrica ( $\mathrm{mS} \mathrm{cm}^{-1}$, conductimetría) & 0,18 \\
\hline Capacidad de intercambio catiónico ( $\mathrm{cmol} \mathrm{kg}^{-1}$, espectrofotometría de emisión) & 17,4 \\
\hline Porcentaje de sodio intercambiable (\%, espectrofotometría de emisión) & 0,34 \\
\hline Sulfato extraíble 1:2,5 ( $\mathrm{mg} \mathrm{kg}^{-1}$, turbidimetría) & 20,7 \\
\hline Relación $\mathrm{Ca} / \mathrm{Mg}$ (espectrofotometría de emisión) & 4,71 \\
\hline Relación $\mathrm{K} / \mathrm{Mg}$ (espectrofotometría de emisión) & 0,52 \\
\hline Zinc ( $\mathrm{mg} \mathrm{kg}^{-1}$, espectroscopia de absorción atómica) & 3,97 \\
\hline Cobre (mg kg-1 , espectroscopia de absorción atómica ) & 6,12 \\
\hline Manganeso ( $\mathrm{m} \mathrm{kg}^{-1}$, espectroscopia de absorción atómica) & 377 \\
\hline Hierro ( $\mathrm{mg} \mathrm{kg}^{-1}$, espectroscopia de absorción atómica) & 169 \\
\hline
\end{tabular}


plantas ha-1 $(1 \mathrm{~m}$ por $0,5 \mathrm{~m})$. Cada subparcela se dividió en dos sub-subparcelas con los clones Barrett y Yaguareté (tercer factor). Los bloques, parcelas, subparcelas y subsubparcelas fueron separados por un borde del clon Salix alba $\mathrm{x}$ Salix babylonica L. 'Ragonese 131-25 INTA'. Los muestreos se llevaron a cabo sobre las dos filas y las 12 hileras centrales de cada sub-subparcela $(n=24$ plantas).

Se utilizó un polietileno negro de $100 \mu \mathrm{m}$ de espesor como mulching para controlar las malezas. En las parcelas regadas, se instaló una cinta de riego por goteo en forma paralela a cada fila de plantación por debajo del mulching plástico. La plantación se realizó entre el 28 de agosto y el 1 de septiembre de 2012, en forma manual con estacas de $70 \mathrm{~cm}$ de longitud. Las plantas muertas fueron repuestas por única vez en el segundo año. Se aplicó Sulfluramida 0,3\% para el control de hormigas y Carbaryl $8 \%$ para controlar el crustáceo isópodo Armadillidium vulgare Latreille.

Datos climáticos. La temperatura y precipitación fueron registradas por una estación meteorológica automática (Davis Instruments) situada a $200 \mathrm{~m}$ del ensayo (figura 1). El equipo de riego funcionó durante los meses con balance hídrico potencialmente negativo de acuerdo a los datos históricos (noviembre a mayo, Hurtado et al. 2006). El riego mantuvo el suelo a capacidad de campo, mientras que las parcelas de secano experimentaron los episodios de sequía que ocurrieron durante el periodo de crecimiento. La lámina de riego aportada fue de $543 \mathrm{~mm}$ en la temporada 2013 - 2014, y 843 mm durante 2014 - 2015.

Determinación de la biomasa. La cosecha se llevó a cabo manualmente durante el período de reposo en invierno en dos etapas. Primero, se extrajeron todas las guías de tres cepas madre de cada sub-subparcela, determinando su peso fresco, el peso seco a $105^{\circ} \mathrm{C}$ y la relación peso seco/ peso fresco. Luego, se cosecharon todas las guías de las 21 plantas restantes de cada sub-subparcela y su peso fresco determinado con un dinamómetro. Este valor se transformó en peso seco utilizando la relación calculada previamente. El rendimiento se estimó extrapolando la biomasa seca de cada parcela a una hectárea.

Análisis estadístico. Los análisis de varianza (ANDEVA) fueron realizados utilizando el programa INFOSTAT (InfoStat 2008). Se utilizó un modelo para bloques con parcelas divididas que incluyó el riego, la densidad y el clon como factores. Debido al diseño utilizado los factores presentan restricciones a la aleatorización. El análisis post-hoc se llevó a cabo utilizando la prueba de Tukey $(P<0,05)$.

\section{RESULTADOS}

Los rendimientos de las parcelas regadas fueron significativamente superiores al tratamiento de secano en ambas temporadas de crecimiento, independientemente de la densidad y el clon (cuadro 2 y figura 2). En la temporada 2013-2014, el rendimiento promedio de las parcelas regadas varió entre 20,1 y 22,6 $\mathrm{Mg} \mathrm{ha}^{-1}$ año $^{-1}$, mientras que las parcelas de secano rindieron entre 15,6 y $17,6 \mathrm{Mg} \mathrm{ha}^{-1}$ año $^{-1}$. En la temporada 2014-2015 la biomasa producida por las parcelas regadas varió entre 18 y $20,2 \mathrm{Mg} \mathrm{ha}^{-1} \mathrm{año}^{-1}$, mientras que el rango en secano fue de 11,2 a $13,4 \mathrm{Mg} \mathrm{ha}^{-1}$ año $0^{-1}$. Estos resultados sugieren que la cantidad de agua recibida durante la temporada de crecimiento fue el factor principal que afectó el rendimiento. En consecuencia, se llevó a cabo una regresión entre la biomasa seca producida

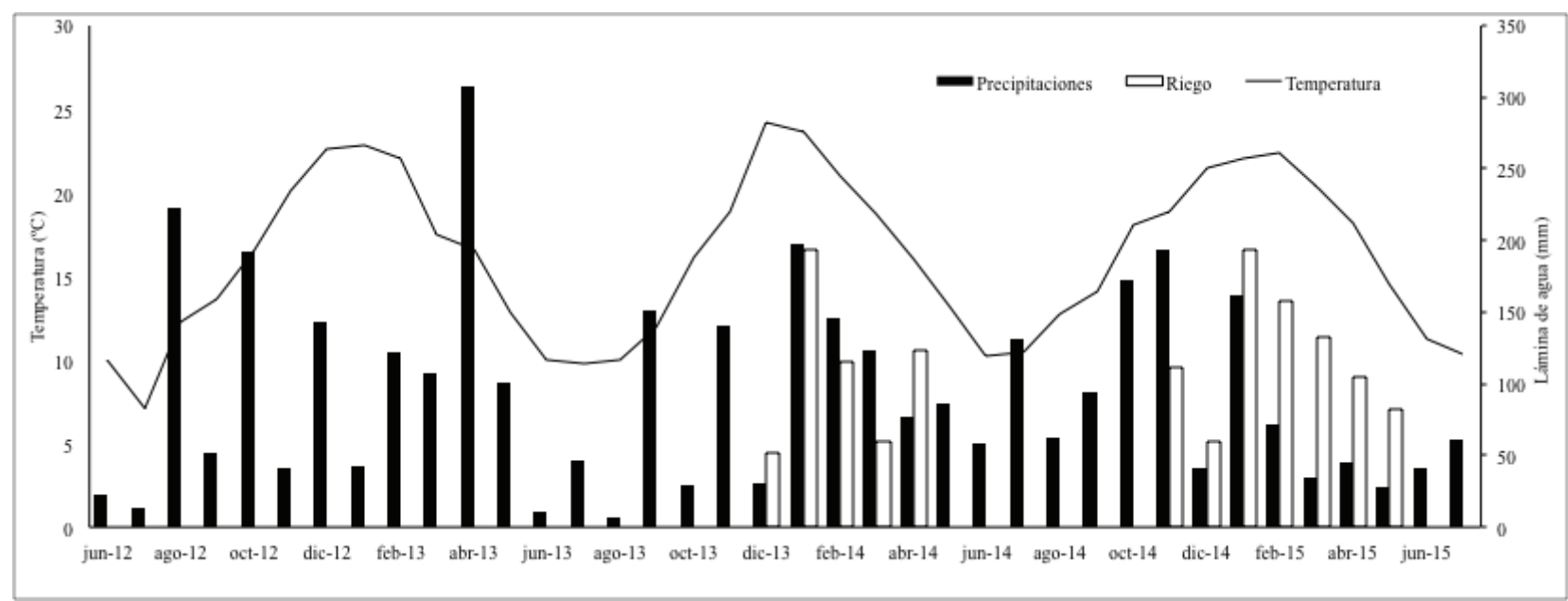

Figura 1. Temperatura media mensual, precipitaciones y lámina de riego suministrada durante el período del ensayo. Las precipitaciones y el riego son los valores acumulados mensuales.

Monthly mean temperature, rainfall and irrigation values during the experiment. Rainfall and irrigation are the accumulated monthly values. 
y el aporte de agua, sumando las precipitaciones y el riego (figura 3). El aporte de agua explica el $92 \%$ de la variabilidad de la biomasa seca en Barrett, y el $83 \%$ en Yaguareté, confirmando que este factor fue el más determinante en las condiciones de este trabajo.

\section{DISCUSIÓN}

La ausencia de diferencias significativas en el rendimiento de biomasa seca entre las distintas densidades de plantación del presente estudio es similar a lo reportado previamente para sauce por Willebrand et al. (1993). Es probable que con rotaciones más largas, haya un aumento de la competencia entre cepas en las densidades más

Cuadro 2. Valores de $P$ de los factores del modelo ANDEVA de la biomasa seca $\left(\mathrm{Mg} \mathrm{ha}^{-1}\right.$ año $\left.^{-1}\right)$ producida en las dos temporadas de muestreo. $* P<0,05 ; * * P<0,01$.

Analyses of variance for the dry biomass $\left(\mathrm{Mg} \mathrm{ha}^{-1}\right)$ produced during the two years of sampling (2013-2014 and 2014-2015). $P$ values for each factor are indicated in the table. Significance levels: $*=P<0.05$; $* *=P<0.01$.

\begin{tabular}{ccc}
\hline Factores & Biomasa & Biomasa \\
& $2013-2014$ & $2014-2015$ \\
\hline Riego & $0,0029 * *$ & $0,0122 *$ \\
Densidad & 0,7731 & 0,7806 \\
Clon & 0,2676 & 0,0972 \\
\hline
\end{tabular}

elevadas que disminuya el crecimiento, pero con rotaciones de un año no se detectaron diferencias. Según estos resultados, sería conveniente plantar a menor densidad, ya que reduciría los costos de implantación que son elevados en este tipo de sistemas (Keoleian y Volk 2005, Baettig et al. 2010) y se evitaría la competencia entre cepas en rotaciones más largas. En cambio, al analizar la productividad en función del riego las diferencias encontradas son significativas. Los tratamientos con riego rinden más que los tratamientos de secano, independientemente del clon y la densidad de plantación, y la disponibilidad de agua explica la mayor parte de la variabilidad en la biomasa observada a lo largo de los dos años para ambos clones. Estos resultados son similares a los publicados para una red de parcelas experimentales de SRC en Italia, en los que se identificó a la disponibilidad de agua como el factor determinante en la acumulación de biomasa (Bergante et al. 2010). Los valores de productividad de las parcelas con riego complementario representarían un valor cercano al rendimiento potencial máximo alcanzable para un sistema de SRC con sauces en la zona, ya que el contenido de nutrientes del suelo es elevado y el mulching plástico ejerce un eficiente control sobre las malezas (Böhlenius y Övergaard 2015).

A pesar de que los clones analizados no fueron seleccionados específicamente para cultivo bioenergético, la productividad anual en los tratamientos se secano fue comparable a la reportada para otros sistemas SRC en condiciones similares. Para el Reino Unido, se han reportado valores máximos de 12-14 $\mathrm{Mg} \mathrm{ha}^{-1}$ año $^{-1}$ de materia seca al final de la rotación para cuatro genotipos de sauce (Cunniff et al. 2015). Karp et al. (2011) indican rendimientos
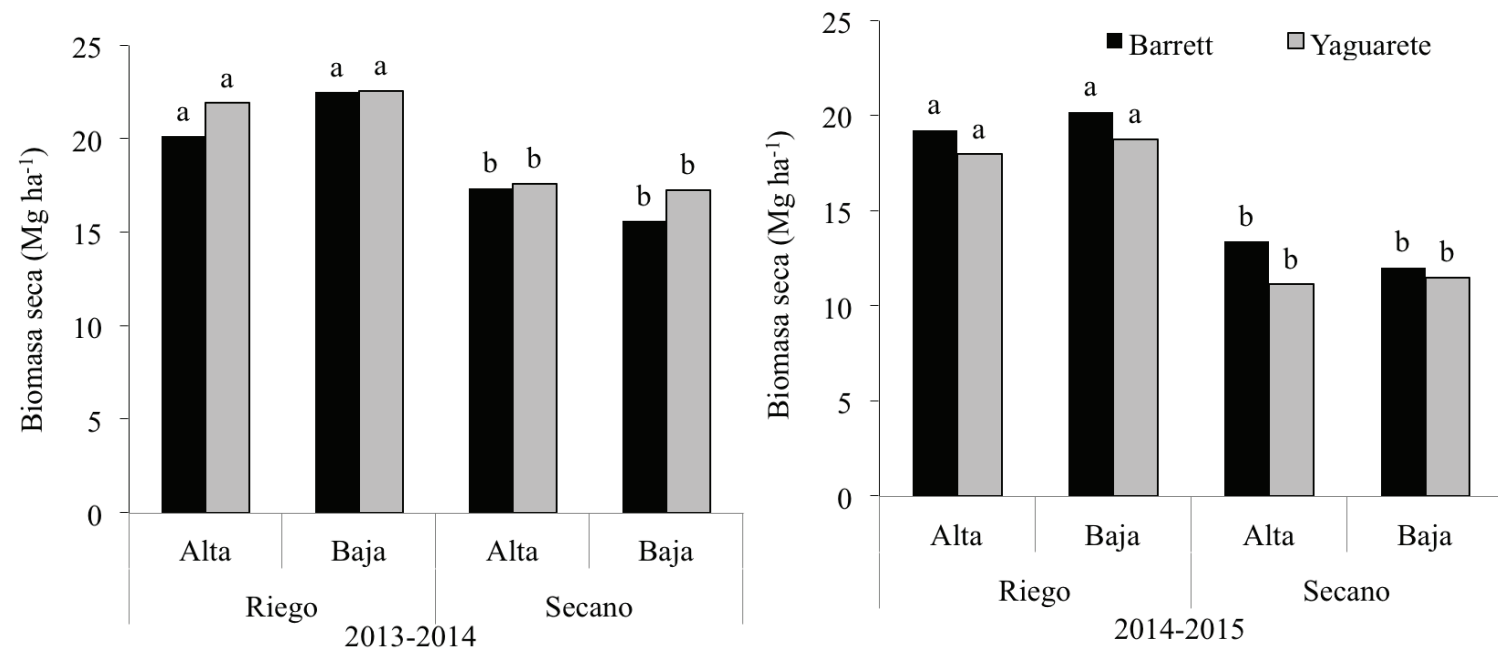

Figura 2. Biomasa seca $\left(\mathrm{Mg} \mathrm{ha}^{-1}\right)$ en las temporadas 2013 - 2014 y 2014 - 2015, para $n=24$. Las medias con de la misma letra no difieren significativamente (prueba de Tukey $P<0,05$ ) para el factor riego. Barras verticales: desvío estándar. Alta: densidad de plantación 20.000 plantas ha ${ }^{-1}$. Baja: densidad de plantación 13.333 plantas ha ${ }^{-1}$.

Dry biomass $\left(\mathrm{Mg} \mathrm{ha}^{-1}\right)$ during the first year (2013-2014) and the second year of harvesting (2014-2015) for $\mathrm{n}=24$. Means followed by the same letter did not differ (Tukey`s test $P<0.05$ ) for the irrigation factor. Vertical bars: standard deviation. Alta: density 20,000 plants ha ${ }^{-1}$. Baja: density 13,333 plants ha ${ }^{-1}$. Riego: irrigation treatment. Secano: rainfed treatment. 


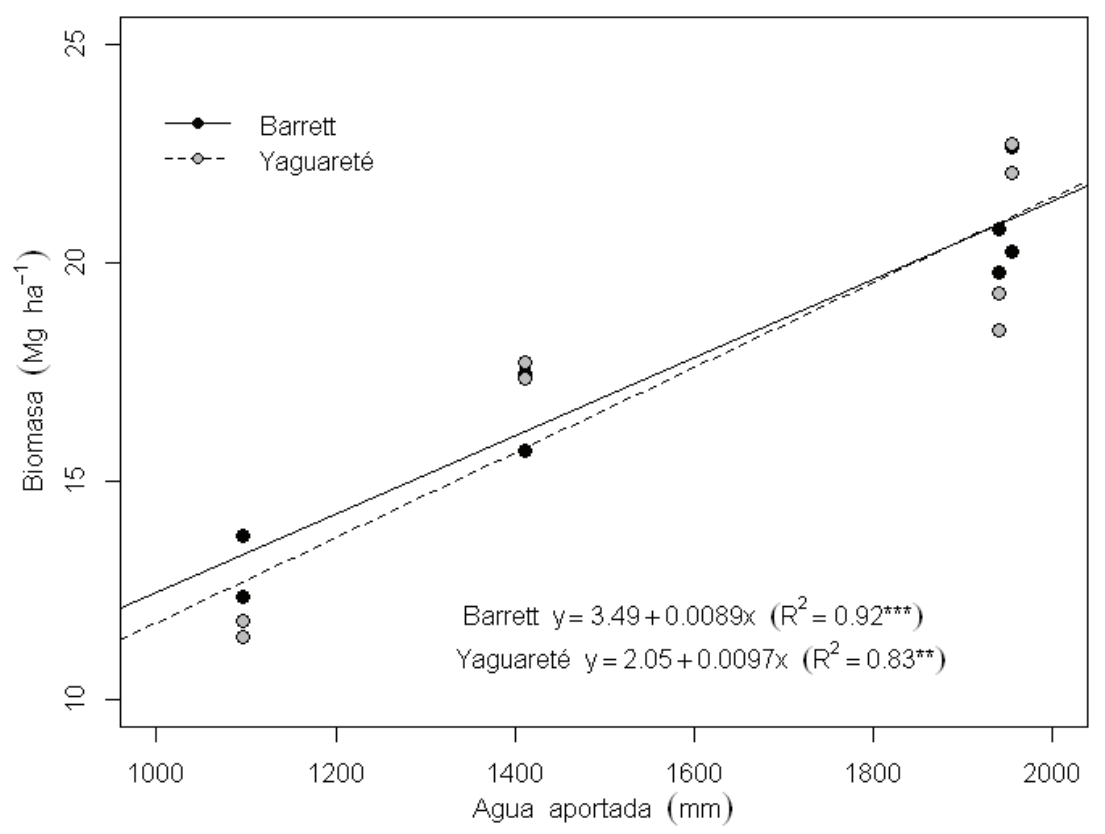

Figura 3. Relación entre la biomasa seca producida y el agua aportada al sistema (como precipitaciones y riego) para todos los años y tratamientos. El valor de $R^{2}$ es el ajustado $(n=16)$. ** $P<0,001 ; * * * P<0,0001$.

Relationship between dry biomass and water supply (rainfall plus irrigation) for all years and treatments. $\mathrm{R}^{2}$ values are the adjusted ones $(n=16)$.

promedio anuales de $14 \mathrm{Mg} \mathrm{ha}^{-1}$ año $^{-1}$ de materia seca para genotipos de sauce mejorados para producir biomasa. Teniendo en cuenta que en este ensayo se cosecha cada año, existe la posibilidad de que con rotaciones más largas se alcancen productividades mayores (Aylott et al. 2008, Quaye y Volk 2013). En el caso del tratamiento con riego, la productividad de este ensayo es menor a la reportada de $28 \mathrm{Mg} \mathrm{ha}^{-1}$ año $^{-1}$ de materia seca para un sistema de SRC bajo riego con álamo en Mendoza, Argentina (Bustamante et al. 2011). Una diferencia importante es que el ensayo mencionado tuvo una rotación más larga (dos años) que explicaría los mayores rendimientos observados.

Si bien los resultados preliminares son prometedores, es necesario analizar la evolución de la productividad de estos genotipos a lo largo de las sucesivas rotaciones, que dependerá del mantenimiento de la capacidad de rebrote y de la supervivencia de las cepas (Karp et al. 2011). Esta información es relevante para determinar por cuánto tiempo serán productivas las plantaciones y estimar la rentabilidad comercial de los sistemas SRC.

\section{CONCLUSIONES}

La hipótesis planteada en este trabajo solo se verificó parcialmente. La disponibilidad de agua es el principal factor que determina el rendimiento en biomasa seca, mientras que el genotipo y la densidad de plantación no tienen un efecto significativo en las condiciones de este ensayo.

\section{AGRADECIMIENTOS}

A T. Cerrillo por proporcionar el clon Yaguareté y al Dr. J. Di Rienzo por su ayuda con el programa INFOSTAT. A P. Etchevers, M. Bartolozzi, S. Martínez y J. Vera Bahima por la ayuda técnica. G. Doffo y M.E. Rodríguez son becarios de CONICET. V. Luquez es investigadora de CONICET. El proyecto fue financiado por el PIA 10007-Ministerio de Agroindustria, Argentina.

\section{REFERENCIAS}

Aylott MJ, E Casella, I Tubby, NR Street, G Taylor. 2008. Yield and spatial supply of bioenergy poplar and willow short rotation coppice in the UK. New Phytologist 178: 358-370. DOI: 10.1111/j.1469-8137.2008.02396.x

Baettig R, M Yáñez A, M Albornoz A. 2010. Cultivos dendroenergéticos de híbridos de álamo para la obtención de biocombustibles en Chile: estado del arte. Bosque 32(2): 89-99.

Bergante S, G Facciotto, G Minotta. 2010. Identification of the main site factors and management intensity affecting the Htablishment of Short-Rotation-Coppices (SRC) in Northern Italy through stepwise regression analysis. Central European Journal of Biology 5(4): 522-530. DOI: 10.2478/ s11535-010-0028-y

Bustamante J, D Funes, M Clausen, M Barbeito. 2011. Populus $x$ canadensis Conti 12 como fuente de energía. Tercer Congreso Internacional de Salicáceas en Argentina. Consultado 18 oct. 2016. Disponible en http://jornadasdesalica- 
ceas2011.blogspot.com.ar/p/trabajos-tecnicos.html.

Böhlenius H, R Övergaard. 2015. Exploration of optimal agricultural practices and seedling types for establishing poplar plantations. Forests 6: 2785-2798. DOI:10.3390/f6082785.

Cerrillo T, ME Rodríguez, FG Achinelli, GN Doffo, V Luquez. 2013. Do greenhouse experiments predict willow responses to long term flooding events in the field? Bosque 34(1): 71-79. DOI: $10.4067 /$ S0717-92002013000100009.

Cerrillo T. 2014. Selección de 6 nuevos clones de sauce (Salix spp.) para el Delta del Paraná. Jornadas de Salicáceas 2014, La Plata, Argentina. Consultado 2 may. 2017. Disponible en http://jornadasdesalicaceas2014.blogspot.com.ar/p/actas-de-las-jornadas-de-salicaceas-2014.html

Cunniff J, SJ Purdy, TJP Barraclough, M Castle, AL Maddisson, LE Jones, IF Shield, AS Gregory, A Karp. 2015. High yielding biomass genotypes of willow (Salix spp.) show differences in below ground biomass allocation. Biomass \& Bioenergy 80:114-127. DOI: http://dx.doi.org/ 10.1016/j.biombioe.2015.04.020

Doffo G, S Monteoliva, ME Rodríguez, V Luquez. 2017. Physiological responses to alternative flooding and drought stress episodes in two willow (Salix spp.) clones. Canadian Journal of Forest Research 47(2): 174-182. DOI: dx.doi. org/10.1139/cjfr-2016-0202

Hurtado MA, JE Giménez, MG Cabral. 2006. Análisis ambiental del partido de La Plata. Aportes al ordenamiento territorial. Buenos Aires, Argentina. Consejo Federal de Inversiones. $134 \mathrm{p}$.

InfoStat. 2008. Manual de usuario, Versión 2008. Grupo InfoStat, FCA, Córdoba, Argentina. Universidad Nacional de
Córdoba. 334 p.

Karp A, SJ Hanley, SO Trybrush, W Macalpine, M Pei, I Shield. 2011. Genetic improvement of willow for bioenergy and biofuels. Journal of Integrative Plant Biology 53(2): 151165. DOI: 10.1111/j.1744-7909.2010.01015.x

Keoleian GA, TA Volk. 2005. Renewable energy from willow biomass crops: life cycle energy, environmental and economic performance. Critical Reviews in Plant Science 24: 385-406. DOI: 10.1080/07352680500316334

Ministerio de Energía y Minería. 2016. Balances Energéticos 2014. Consultado 6 may. 2016. Disponible en http://www. energia.gob.ar

Quaye AK, TA Volk. 2013. Biomass production and soil nutrients in organic and inorganic fertilized willow biomass production systems. Biomass \& Bioenergy 57: 113-125. DOI: http://dx.doi.org/10.1016/j.biombioe.2013.08.002

Volk TA, LP Abrahamson, CA Nowak, LB Smart, PJ Tharakan, EH White. 2006. The development of short- rotation willow in the northeastern United States for bionergy and bioproducts, agroforestry and phytoremediation. Biomass \& Bioenergy 30: 715-727. DOI:10.1016/j.biombioe.2006.03.001.

Wikberg J, E Ögren. 2007. Variation in drought resistance, drought acclimation and water conservation in four willow cultivars used for biomass production. Tree Physiology 27:1339-1346.

Willebrand E, S Ledin, T Verwijst. 1993. Willow coppice systems in short rotation forestry: effects of plant spacing, rotation length and clonal composition on biomass production. Biomass \& Bioenergy 4: 323-331. 